\title{
Evidencia de autopoliploidía y translocaciones en el cariotipo de Tigridia pavonia (Iridaceae, Iridoideae) de la Reserva Ecológica del Pedregal de San Ángel, México
}

\author{
Evidence of autopolyploidy and translocations in the karyotype of \\ Tigridia pavonia (Iridaceae, Iridoideae) from the Reserva Ecológica del \\ Pedregal de San Ángel, Mexico
}

\author{
Fernando Tapia-Pastranal’2 (10), Fernando Tapia-Aguirrel (D)
}

1 Universidad Nacional Autónoma de México, Facultad de Estudios Superiores Zaragoza, División de Estudios de Posgrado e Investigación Laboratorio de Genecología, Batalla del 5 de Mayo s.n., Col. Ejército de Oriente, 04510 Cd. Mx., México.

2 Autor para la correspondencia: pasfer@unam.mx

\section{Citar como:}

Tapia-Pastrana, F. y F. Tapia-Aguirre. 2017. Evidencia de autopoliploidía y translocaciones en el cariotipo de Tigridia pavonia (Iridaceae, Iridoideae) de la Reserva Ecológica del Pedregal de San Ángel, México. Acta Botanica Mexicana 121: 151-158. DOI: http://dx.doi. org/10.21829/abml21.2017.1205

Recibido: 2 de enero de 2017.

Revisado: 29 de junio de 2017

Aceptado: 7 de julio de 2017.

DOI:

http://dx.doi.org/10.21829/abml21.2017.1205

\section{Resumen:}

Antecedentes y Objetivos: Tigridia pavonia fue propuesto como un taxon alotetraploide $2 n=4 x=28$, pero $\sin$ evidencia citogenética que sustente su origen híbrido. Es una especie de amplia distribución en México y sus poblaciones carecen de una fórmula cariotípica y un análisis detallado de los cromosomas con satélites como criterio para determinar el número de organizadores nucleolares que confirmen o no dominancia nucleolar. En este trabajo se analiza y describe el número y arquitectura cromosómica de T. pavonia de una población mexicana, en búsqueda de evidencias que soporten o descarten su origen híbrido y se propone una fórmula cariotípica acorde al nivel y origen de ploidía.

Métodos: Se utilizó una técnica de extendido en superficie y secado al aire que incluye maceración enzimática y choque hipotónico en meristemos radiculares para obtener los cromosomas en mitosis de seis individuos de $T$. pavonia, nativa de la Reserva Ecológica del Pedregal de San Ángel, en la Ciudad de México.

Resultados clave: Se confirmó un cariotipo bimodal con 28 cromosomas que, de acuerdo a su similitud morfológica, fueron incluidos en siete grupos de cuatro cromosomas homólogos cada uno. Los cromosomas del grupo más pequeño exhibieron constricciones secundarias asociadas a macrosatélites lo que evidenció ausencia de dominancia nucleolar o amfiplastía diferencial. En el grupo de cromosomas grandes se observaron configuraciones que sugieren rearreglos por translocaciones. Se propone la fórmula $6 \mathrm{~m}+8 \mathrm{sm}$ para el cariotipo haploide. Conclusiones: Citogenéticamente, la presencia de cuatro satélites descarta un origen alotetraploide y la evidencia de posibles translocaciones se correlaciona con fragmentos, cromosomas B y centrómeros frágiles observados en otras especies del género. Lo anterior apoya el papel activo de las translocaciones en la conformación del cariotipo bimodal de T. pavonia.

Palabras clave: ausencia de dominancia nucleolar, autotetraploide, citogenética, rearreglos cromosómicos, satélites.

\section{ABSTRACT:}

Background and Aims: Tigridia pavonia was proposed as an allotetraploid taxon $2 n=4 x=28$, but without cytogenetic evidence supporting its hybrid origin. It is a widespread species in Mexico and its populations lack karyotype and detailed analysis of chromosomes with satellites as a criterion to determine the number of nucleolar organizers that confirm or not nucleolar dominance. In this paper, we analyze and describe the number and chromosome architecture of T. pavonia from a Mexican population in search of evidences that support or discard its hybrid origin and we propose a karyotype according to the level and origin of ploidy.

Methods: A surface spreading and air drying technique was used that includes enzymatic maceration and hypotonic shock in meristem roots to obtain mitotic chromosomes from six individuals of T. pavonia native to the Reserva Ecológica del Pedregal de San Ángel, Mexico City.

Key results: A bimodal karyotype with 28 chromosomes was confirmed, which according to their similar morphology were included in seven groups of four homologous chromosomes each. All chromosomes in the smallest group exhibited secondary constrictions associated to macrosatellites and this shows the absence of nucleolar dominance or differential amphiplasty. Moreover, in the group of largest chromosomes configurations were observed that suggest rearrangements by translocations. The haploid karyotype $6 \mathrm{~m}+8 \mathrm{sm}$ is proposed here.

Conclusions: Cytogenetically, the presence of four satellites discards an allotetraploid origin and the evidence of possible translocations correlates with fragments, B chromosomes and fragile centromeres observed in other species of this genus. This supports the active role of translocations in the conformation of bimodal karyotype of T. pavonia.

Key words: absence of nucleolar dominance, autotetraploid, chromosomal rearrangements, cytogenetics, satellites. 


\section{INTRODUCCIÓN}

El género Tigridia Jussieu pertenece a la familia Iridaceae, subfamilia Iridoideae, tribu Tigridiae, subtribu Tigridiinae (Goldblatt, 1982) y agrupa entre 50 y 55 especies (Goldblatt et al., 2008; The Plant List, 2013) que se distribuyen en México, América Central y los Andes. En México el género está representado por 35 taxa entre especies y variedades, y la nativa Tigridia pavonia (L.f.) DC. es la especie de más amplia distribución (Calderón de Rzedowski, 1987; Henrich y Goldblatt, 1994; López-Ferrari y Espejo, 1994; Rodríguez y Sytsma, 2006). Se le encuentra principalmente en bosques de Pinus y Quercus a lo largo de la Sierra Madre Occidental, la Sierra Madre Oriental, el Eje Volcánico Transversal, la Sierra Madre del Sur y la Sierra Madre de Chiapas (Molseed, 1970) y se considera que México representa su centro de mayor diversidad genética (Arzate-Fernández et al., 2008). Es una hierba criptófita de bulbo carnoso, posee hojas grandes, lanceolares y envainantes, su flor es actinomorfa, posicionada sobre un escapo cilíndrico con varias flores bilabiadas grandes, de color rojo a amarillo y es considerada una de las flo-

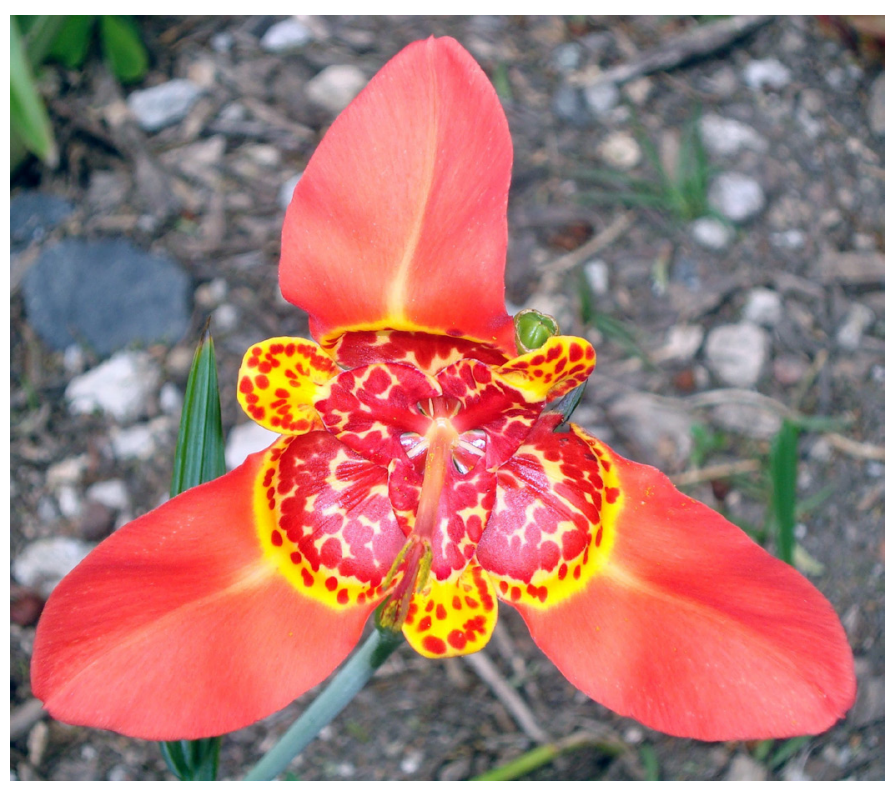

Figura 1: Flor en antesis de Tigridia pavonia (L.f.) DC. con los tépalos totalmente extendidos. res más bellas (Fig. 1) y más representativas de la Reserva Ecológica del Pedregal de San Ángel (REPSA) (Velázquez-López et al., 2009). La REPSA es una reserva natural urbana que pertenece a la Universidad Nacional Autónoma de México (UNAM) y desde el siglo XIX es considerada como un sitio de alta riqueza de especies vegetales (Rzedowski, 1954; Valiente-Banuet y Luna, 1990), animales (Negrete y Soberón, 1994) y hongos (Hernández-Cuevas et al., 2003). Tigridia pavonia es conocida como hierba de la trinidad, cacomit, flor del tigre y oceloxóchitl. Además de ser una especie ornamental también se le utiliza como alimento y, por sus propiedades antipiréticas, en la medicina tradicional (Vázquez et al., 2001; Rojo y Rodríguez, 2002).

Desde el punto de vista citogenético, Goldblatt (1982) sugirió que la subtribu Tigridiinae tiene un número cromosómico básico $x=14$. Posteriormente, Kenton y Heywood (1984) registran una especie de Tigridia colectada en Perú (Tigridia sp.) con un número cromosómico diploide $2 n=14$ y proponen $x=7$ para el género Tigridia. Por otra parte, los estudios citogenéticos realizados en $T$. pavonia incluyen registros de $2 n=26$ (Brittingham, 1934; Sharma y Sharma, 1961) y $2 n=28$ (Sakai, 1952; Molseed, 1970; Goldblatt, 1982; Kenton y Heywood, 1984). Aunque en todos los trabajos anteriores se describen complementos cromosómicos claramente bimodales, no existen propuestas de fórmulas cariotípicas y sólo en dos de éstos se registraron tallas cromosómicas. A las discrepancias en los números cromosómicos obtenidos con técnicas citogenéticas diferentes se suma la propuesta de Kenton y Heywood (1984) sobre el posible origen alotetraploide de $T$. pavonia en función de un número básico $x=7(2 n=4 x=28)$ y no aquel $x=14$ propuesto por Goldblatt (1982).

Los objetivos de la presente investigación son analizar citogenéticamente una muestra de individuos de $T$. pavonia de la REPSA para: (I) corroborar el número cromosómico de este taxon, (II) analizar en detalle su arquitectura cromosómica en la búsqueda de evidencias que descarten o sustenten el origen híbrido con particular énfasis en el número y morfología de los cromosomas con 
satélite como criterio para establecer la existencia o no de dominancia nucleolar o amfiplastía diferencial y (III) proponer una fórmula cariotípica acorde al nivel de ploidía. La información del sistema genético básico de esta especie es necesaria para interpretar su evolución y su amplia distribución y variabilidad morfológica.

\section{Materiales y Métodos}

\section{Colecta de material}

Se recolectaron frutos de Tigridia pavonia durante el mes de noviembre de 2014 en las zonas de amortiguamiento A8 (Biológicas) y A10 (Jardín Botánico) de la Reserva Ecológica del Pedregal de San Ángel (REPSA) de Ciudad Universitaria ( UNAM, 2005), en el sur de la cuenca del valle de México; entre $19^{\circ} 20^{\prime} 2^{\prime \prime}$ y $19^{\circ} 13^{\prime} 45^{\prime \prime} \mathrm{N}, 99^{\circ} 08^{\prime} 26^{\prime \prime}$ y $99^{\circ} 14^{\prime} 3 " \mathrm{O} ; 2250$ a $2350 \mathrm{~m}$ s.n.m.

\section{Germinación de semillas}

Un grupo de semillas seleccionadas al azar, provenientes de seis individuos, fueron puestas a germinar en cajas de Petri con algodón humedecido en agua destilada y cubierto con papel absorbente en incubadora a $32{ }^{\circ} \mathrm{C}$ por la noche y a temperatura ambiente durante el día, por un periodo mayor a tres semanas y hasta su germinación. Las raíces de 1-2 $\mathrm{mm}$ de largo fueron cortadas y pretratadas en 8-hidroxiquinoleína $0.002 \mathrm{M}$ durante $5 \mathrm{~h}$ a temperatura ambiente y en oscuridad. Posteriormente, fueron fijadas en solución Farmer, etanol-ácido acético, 3:1.

\section{Obtención de cromosomas}

Para la obtención de los cromosomas en metafase se siguió el método de extendido en superficie y secado al aire propuesto por Tapia-Pastrana y Mercado-Ruaro (2001), el cual se basa en la maceración enzimática (pectinasa $20 \%+$ celulasa $2 \%$ ) de los meristemos apicales durante $2 \mathrm{~h}$ a $37^{\circ} \mathrm{C}$, seguido de la transferencia de los protoplastos a una solución fresca de $\mathrm{KCl} 0.075$ $\mathrm{M}$ durante $15-20$ minutos a $37^{\circ} \mathrm{C}$. Posteriormente, se realizaron dos lavados con la misma solución y el botón celular fue fijado en solución Farmer. Dos gotas del botón celular se colocaron sobre portaobjetos limpios y la tinción de los cromosomas se realizó con Giemsa a $10 \%$. Las preparaciones se hicieron permanentes empleando resina sintética (Sigma) como medio de montaje. Los mejores campos fueron fotografiados con un microscopio óptico Zeiss Axioskop (Carl Zeiss Jena GmbH, Jena, Alemania), usando película Kodak Technical Pan .

Se utilizó un vernier digital Mitutoyo Digimatic Caliber CD-G'BS (Mitutoyo Corporation, Kanagawa, Japón) para establecer las tallas cromosómicas y otras medidas morfométricas sobre siete fotografías de placas en metafase típica con similar grado de condensación y amplificadas con la misma magnificación.

Para la obtención de la fórmula cariotípica y nomenclatura, se aplicó el sistema propuesto por Levan et al. (1964). La proporción de brazos, $r$, se obtuvo mediante el cociente $\mathrm{q} / \mathrm{p}$, valor que muestra la relación del brazo largo y el brazo corto de los cromosomas individuales. La proporción de la suma total de longitudes de brazos cortos respecto a la suma total de longitudes cromosómicas como indicador de simetría o asimetría de un cariotipo (TF\%) se realizó según Sinha y Roy (1979).

\section{Resultados}

\section{Cuantificación del cariotipo}

Las características cuantitativas del cariotipo de Tigridia pavonia se describen en el cuadro 1, mientras que en el cuadro 2 se resumen otros datos relacionados con los complementos de la misma. Se observaron un total de 224 células en metafase típica. En todas se registró sin ambigüedad un $2 n=28$, los complementos cromosómicos claramente fueron asimétricos y bimodales, conformados por cuatro cromosomas submetacéntricos grandes $(6.84 \pm 0.33 \mu \mathrm{m}) \mathrm{y}$ 24 cromosomas más pequeños con centrómeros medios (metacéntricos) o ligeramente desplazados (submetacéntricos). En estos se apreció que los cuatro cromosomas homólogos más pequeños $(\mathrm{sm})$ portaban constricciones secundarias asociadas a macrosatélites (Cuadro 1; Fig. 2), según la clasificación de Battaglia (1955). 
Cuadro 1: Medidas cromosómicas promedio obtenidas de siete células en metafase típica de Tigridia pavonia (L.f.) DC. GC: grupo cromosómico; LC: longitud cromosómica en $\mu \mathrm{m}$; LBL: longitud de brazo largo en $\mu \mathrm{m}$; LBC: longitud de brazo corto en $\mu \mathrm{m} ; r$ : LBL/LBC; $\mathrm{N}$ : nomenclatura; *: constricción secundaria y satélite.

\begin{tabular}{cccccc}
\hline GC & LC & LBL & LBC & $r$ & $\mathrm{~N}$ \\
\hline $1-4$ & $6.84 \pm 0.33$ & 4.66 & 2.17 & 2.14 & $\mathrm{sm}$ \\
$5-8$ & $3.33 \pm 0.40$ & 1.82 & 1.50 & 1.21 & $\mathrm{~m}$ \\
$9-12$ & $3.07 \pm 0.14$ & 1.82 & 1.37 & 1.32 & $\mathrm{~m}$ \\
$13-16$ & $2.90 \pm 0.08$ & 1.86 & 1.03 & 1.80 & $\mathrm{sm}$ \\
$17-20$ & $2.83 \pm 0.11$ & 1.80 & 1.02 & 1.76 & $\mathrm{sm}$ \\
$21-24$ & $2.56 \pm 0.07$ & 1.45 & 1.10 & 1.31 & $\mathrm{~m}$ \\
$25-28$ & $2.23 \pm 0.01$ & 1.42 & 0.80 & 1.77 & $\mathrm{sm} *$ \\
\hline
\end{tabular}

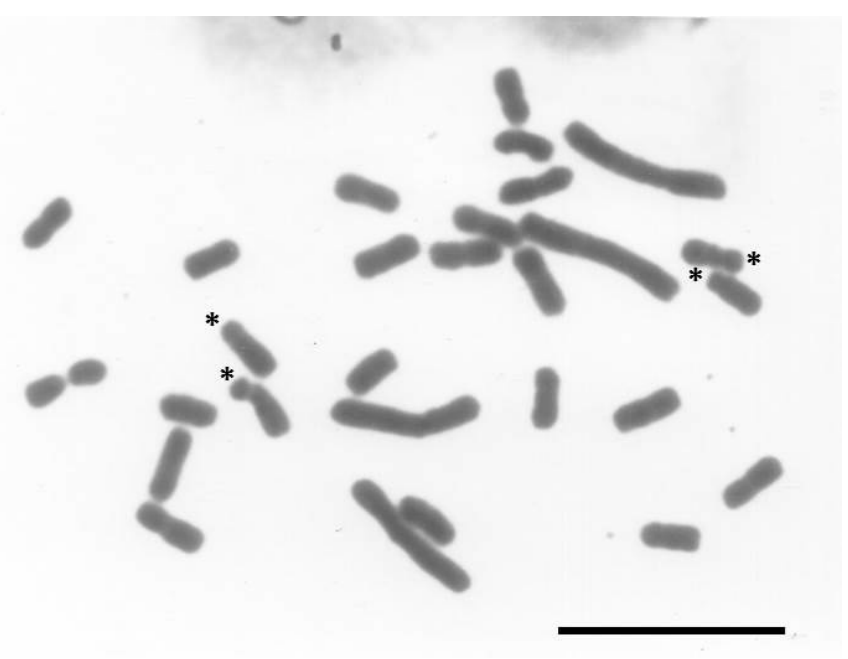

Figura 2: Complemento cromosómico tetraploide $2 n=4 x=28$ de Tigridia pavonia (L.f.) DC. ${ }^{*}=$ cromosomas con satélite. Barra $=10 \mu \mathrm{m}$.

\section{Rearreglos cromosómicos}

Por otra parte, se encontró evidencia de posibles translocaciones particularmente en los cromosomas submetacéntricos grandes donde frecuentemente fueron observados tanto en los brazos cortos y largos segmentos de los cromosomas alineados de manera atípica y en algunos casos formando ángulos raramente observados en cromosomas vegetales (Fig. 3). Asimismo, se observó con relativa frecuencia por lo menos un cromosoma metacéntrico pequeño (Fig. 3) que exhibió un centrómero notoriamente laxo el cual separa ampliamente a ambos brazos (Figs. 3, 4).

\section{Propuesta de fórmula cariotípica}

Para la descripción del cariotipo de T. pavonia de la población bajo estudio se consideró un número básico $x=7$ (Kenton y Heywood, 1984) y sobre la base de la longitud cromosómica y proporción de brazos fue posible caracterizar siete grupos de cuatro cromosomas cada uno con una morfología similar correspondientes a una especie de origen autotetraploide (Cuadro 1). En consecuencia, la fórmula cariotípica haploide propuesta para la población bajo estudio fue $6 \mathrm{~m}+8 \mathrm{sm}$ (Cuadro 2). La figura 5 muestra el cariotipo de T. pavonia donde los cromosomas fueron agrupados por homología, alineados por el centrómero y en orden decreciente, conforme a la propuesta de Kenton y Heywood (1984). No se encontró evidencia de fragmentos ni se observaron cromosomas B.

\section{DISCUSIÓN}

El número cromosómico $2 n=28$ obtenido en la presente investigación para la población estudiada se obtuvo a partir del recuento de 224 células en metafase y por tan-

Cuadro 2: Características cuantitativas del complemento cromosómico de Tigridia pavonia (L.f.) DC. obtenidos a partir de siete células en metafase. FC: fórmula cromosómica; LCTH: longitud cromosómica total haploide en $\mu \mathrm{m}$; TCP: talla cromosómica promedio en $\mu$ m; Rango (longitud del cromosoma mayor-longitud del cromosoma menor); Razón (cromosoma mayor/cromosoma menor); TF\%: índice de asimetría (longitud total de brazos cortos/longitud cromosómica total $\times 100$ ).

\begin{tabular}{ccccccc}
\hline $2 n$ & FC & LCTH & TCP & Rango & Razón & TF\% \\
\hline 28 & $6 \mathrm{~m}+8 \mathrm{sm}$ & $47.54 \pm 1.69$ & $3.39 \pm 0.12$ & $4.61 \pm 0.34$ & $3.06 \pm 0.16$ & 37.83 \\
\hline
\end{tabular}




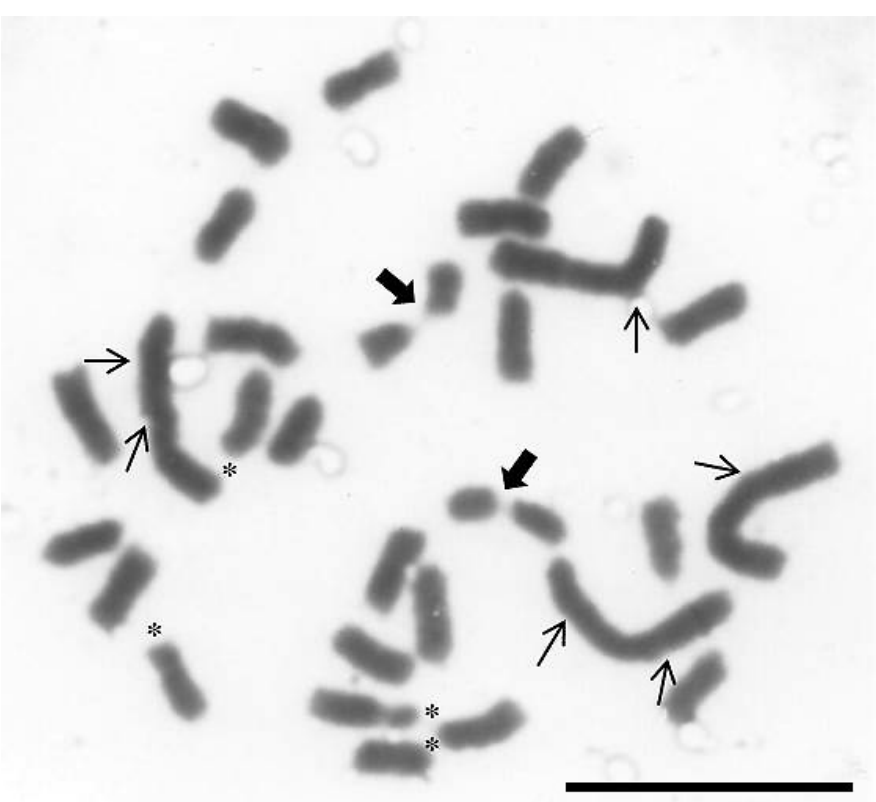

Figura 3: Metafase mitótica de Tigridia pavonia (L.f.) DC. $2 n=4 x=28$. Las flechas estrechas señalan sitios de probables translocaciones. Las flechas gruesas resaltan a dos cromosomas con centrómero laxo. * $=$ cromosomas con satélite. Barra $=10 \mu \mathrm{m}$.

to se corrobora un $2 n=28$ encontrado por Sakai (1952), Goldblatt (1982) y Kenton y Heywood (1984) quienes utilizaron técnicas estándar de squash y tinción Feulgen y corresponde al de una población tetraploide con un número básico $x=7$, reconocido como el predominante en las monocotiledóneas (Raven, 1975; Dahlgren et al., 1985).

La propuesta sobre la participación de translocaciones en la conformación del cariotipo actual de T. pavonia

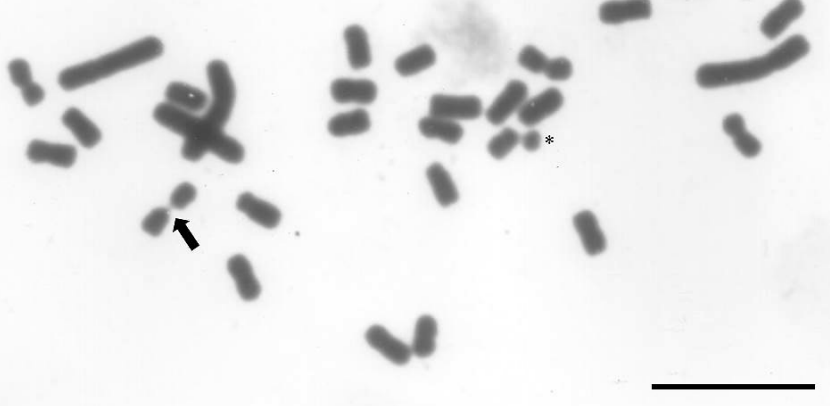

Figura 4: Metafase mitótica de Tigridia pavonia (L.f.) DC. $2 n=4=28$. *=cromosomas con satélite. La flecha gruesa resalta a un cromosoma con centrómero laxo. Barra $=10 \mu \mathrm{m}$.

de la REPSA se sustenta en la identificación de segmentos cromosómicos con alineación atípica a lo largo de ambos brazos de los cromosomas grandes y que frecuentemente se corresponden con inflexiones raramente observadas en los cromosomas de especies vegetales (Fig. 3). Este fenómeno parece correlacionar con los fragmentos y cromosomas B observados en diversas especies de Tigridia (Molseed, 1970) y con el registro de centrómeros frágiles y aparentes constricciones secundarias en los cromosomas grandes de una especie diploide $2 n=14$ descrita como Tigridia sp. (Kenton y Heywood, 1984), en donde además solo se registró un par de cromosomas pequeños con satélites. Tomadas en conjunto, estas observaciones apuntan hacia un papel activo de translocaciones subsecuentes a

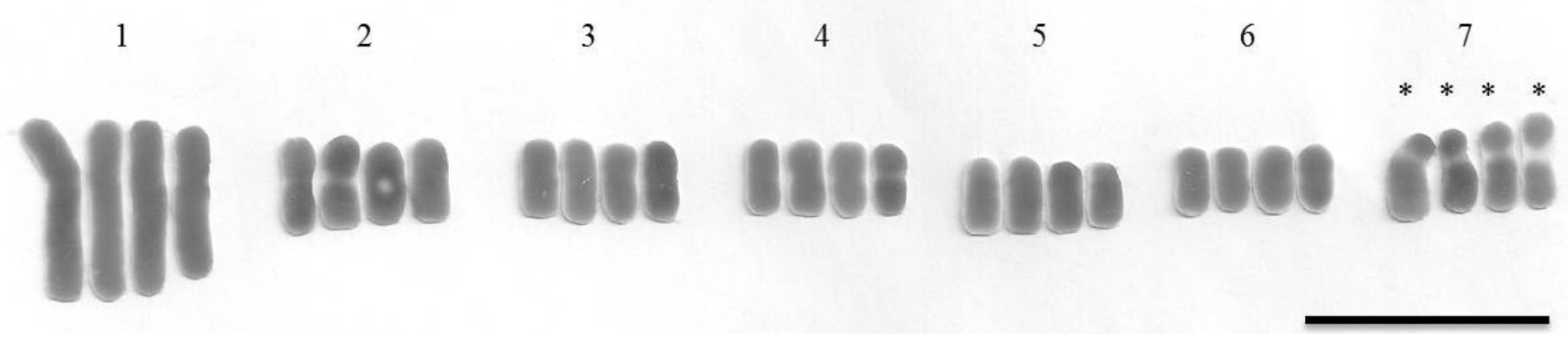

Figura 5: Cariotipo de Tigridia pavonia (L.f.) DC. $(2 n=4 x=28)$, con siete grupos de cuatro cromosomas cada uno con una morfología similar sobre la base de la longitud cromosómica y proporción de brazos. Los cromosomas fueron agrupados por homología, alineados por el centrómero y en orden decreciente. $*=$ cromosomas con satélite. Barra $=10 \mu \mathrm{m}$. 
rompimientos en sitios frágiles en los cromosomas grandes de T. pavonia, fenómeno similar al exhibido por Milla biflora Cav. (Asparagaceae), otra monocotiledónea con amplia distribución (Tapia-Pastrana, 2014) y confirman la opinión de Stebbins (1971), quien sugirió el papel relevante de las translocaciones en la conformación de los cariotipos bimodales de las monocotiledóneas.

Por otra parte, Kenton y Heywood (1984) señalan que existe poca heteromorfia entre los cromosomas grandes de T. pavonia; sin embargo, esta misma observación puede aplicarse a los agrupamientos de los cromosomas pequeños propuestos por las mismas autoras como se aprecia en la figura $4 \mathrm{~b}$ de su investigación. Estos datos parecen describir mejor a una especie de origen autopoliploide y no a una alopoliploide como fue sugerido. Asimismo, la identificación de siete grupos de cuatro cromosomas cada uno con morfología similar obtenidos en la presente investigación refuerza la idea anterior.

Por otro lado, la presencia de satélites ubicados en los brazos cortos de los cuatro cromosomas más pequeños apunta hacia la ausencia de amfiplastía o dominancia nucleolar, es decir la inactivación uniparental de transcripción de los genes del rRNA por competencia de los organizadores nucleolares (Navashin, 1928; Preuss y Pikaard, 2007). A este respecto conviene mencionar que en el proceso autopoliploidía el número de satélites presentes en una especie diploide también se duplica y no involucra pérdida o supresión de la función nucleolar. Las regiones del organizador nucleolar (NOR) asociadas a las constricciones secundarias se muestran laxas y por tanto los satélites se aprecian claramente. Por ejemplo, Medicago sativa L., un reconocido autotetraploide, exhibe cuatro macrosatélites en células en metafase (Falistocco, 1987). En contraparte, las plantas de origen alopoliploide experimentan inactivación de las regiones NOR de uno de los genomas parentales, silenciadas por efecto de dominancia nucleolar y en consecuencia se registra un número menor de satélites. Así, en el complejo Hordeum murinum L. (Poaceae, Triticeae) los citotipos tetraploides y hexaploides surgidos por hibridación exhiben únicamente un par de cromosomas con constric- ciones secundarias y satélites (Cuadrado et al., 2013). De hecho, la inactivación de genes ribosomales es uno de los fenómenos más comunes en miembros híbridos y poliploides de Triticeae (Cermeño y Lacadena, 1985; Carmona et al., 2016).

Así, la identificación de siete grupos cromosómicos de morfología similar y la ausencia de dominancia nucleolar apoyan nuestra propuesta sobre el origen autotetraploide de T. pavonia. Si bien la morfología de los cromosomas en metafase mitótica se ha usado frecuentemente como criterio para distinguir entre autopoliploides y alopoliploides, es necesario emplear el mayor número de evidencias posibles en la determinación de la naturaleza poliploide en especies vegetales (Stebbins, 1971), por lo que en un futuro se deberán realizar estudios meióticos y técnicas de bandeo que apoyen la propuesta anterior.

Asimismo en la población aquí estudiada las tallas cromosómicas se ubicaron en el intervalo de 2.23 hasta $6.84 \mu \mathrm{m}$ el cual concuerda en general con aquellas registradas previamente por Sharma y Sharma (1961), quienes obtuvieron un intervalo de 2.1-8.8 $\mu \mathrm{m}$ y con el de Kenton y Heywood (1984) que lo ubicaron entre 3 y $6.5 \mu \mathrm{m}$. Las diferencias se atribuyen a que T. pavonia contiene un grupo de cromosomas grandes que varían en su grado de contracción durante la metafase mitótica.

\section{CONTRIBUCIÓN DE LOS AUTORES}

FTP dirigió el diseño de la presente investigación, la colecta de semillas, encabezó los ensayos para la obtención de los cromosomas mitóticos, y la redacción del manuscrito. FTA participó en la preparación y análisis del material de trabajo, en la adquisición de datos y en su interpretación. FTP y FTA contribuyeron a la discusión, revisión y aprobación del manuscrito final.

\section{AgradeCIMIENTOS}

Los autores agradecen los comentarios de un revisor anónimo quien mejoró el manejo de la información y clarificación de los resultados. A la División de Estudios de Posgrado e Investigación de la FES-Zaragoza por el apoyo prestado para la realización de la presente investigación. 


\section{FINANCIAMIENTO}

El estudio no tuvo financiamiento por parte de ningún proyecto.

\section{LITERATURA CITADA}

Arzate-Fernández, A. M., A. Hoyos-Basurto, L. M., VázquezGarcía y M. G. Gutiérrez-Martínez. 2008. Caracterización isoenzimática de nueve variedades botánicas de Tigridia pavonia (L.f.) DC. Agrociencia 42(5): 519-528.

Battaglia, E. 1955. Chromosome morphology and terminology (with 12 figures). Caryologia 8(1): 179-187. DOI: http:// dx.doi.org/10.1080/00087114.1955.10797556

Brittingham, Wm. H. 1934. Cytological studies on some genera of the Iridaceae. American Journal of Botany 21(2): 77-83.

Calderón de Rzedowski, G. 1987. Tigridia martinezii, una especie nueva de iridáceas del estado de Hidalgo (México). Boletín de la Sociedad Botánica de México 47: 3-6.

Carmona, A., A. de Bustos, N. Jouve y A. Cuadrado. 2016. Allopolyploidy and the complex phylogenetic relationships within the Hordeum brachyantherum taxon. Molecular Phylogenetics and Evolution 97: 107-119. DOI: http://dx.doi.org/10.1016/j.ympev.2016.01.001

Cermeño, M. C. y J. R. Lacadena. 1985. Nucleolar organizer competition in Aegilops-rye hybrids. Canadian Journal of Genetics and Cytology 27(4): 479-483. DOI: http:// dx.doi.org/10.1139/g85-070

Cuadrado, A., A. Carmona y N. Jouve. 2013. Chromosomal characterization of the three subgenomes in the polyploids of Hordeum murinum L.: New insight into the evolution of this complex. PLoS ONE 8(12): e81385. DOI: http:// dx.doi.org/10.1371/journal.pone.0081385

Dahlgren, R. M. T., H. T. Clifford y P. F. Yeo. 1985. The families of monocotyledons: structure, evolution and taxonomy. Springer Verlag. Berlin, Germany. 520 pp. DOI: http:// dx.doi.org/10.1007/978-3-642-61663-1

Falistocco, E. 1987. Cytogenetic investigations and karyological relationships of two Medicago: M. sativa L. (Alfalfa) and M. arborea L. Caryologia 40(4): 339-346. DOI: http:// dx.doi.org/10.1080/00087114.1987.10797836

Goldblatt, P. 1982. Chromosome cytology in relation to suprageneric systematics of Neotropical Iridaceae.
Systematic Botany 7(2): 186-198. DOI: http://dx.doi. org $/ 10.2307 / 2418327$

Goldblatt, P., A. Rodríguez, M. P. Powell, T. J. Davies, J. C. Manning, M. van der Bank y V. Savolainen. 2008. Iridaceae 'Out of Australasia'? Phylogeny, Biogeography, and Divergence Time Based on Plastid DNA Sequences. Systematic Botany 33(3): 495-508. DOI: http://dx.doi. org/10.1600/036364408785679806

Henrich, J. E. y P. Goldblatt. 1994. Iridaceae. In: Davidse, G., M. Sousa y A. Q. Charter (eds.). Flora Mesoamericana. Volumen 6. Instituto de Biología, Universidad Nacional Autónoma de México. México, D.F., México. Pp. 7180.

Hernández-Cuevas, L., S. Castillo-Argüero, P. GuadarramaChávez, Y. Martínez-Orea, M. A. Romero-Romero y I. Sánchez-Gallén. 2003. Hongos micorrizógenos arbusculares del Pedregal de San Ángel. Las prensas de Ciencias, Universidad Nacional Autónoma de México. México, D.F., México. 82 pp.

Kenton, A. y C. A. Heywood. 1984. Cytological studies in South American Iridaceae. Plant Systematics and Evolution 146(2): 87-104. DOI: http://dx.doi.org/10.1007/ BF00984056

Levan, A., K. Fredga y A. A. Sandberg. 1964. Nomenclature for centromeric position on chromosomes. Hereditas 52(2): 201-220. DOI: http://dx.doi. org/10.1111/j.1601-5223.1964.tb01953.x

López-Ferrari, A. R. y A. Espejo. 1994. Tigridia estelae (Iridaceae: Tigridieae), a new species from Durango, Mexico. Novon 4(4): 386-390. DOI: http://dx.doi. org/10.2307/3391449

Molseed, E. 1970. The genus Tigridia (Iridaceae) of Mexico and Central America. University of California Publications in Botany 54: 1-113.

Navashin, M. S. 1928. Amphiplastie-eine neue karyologische Erscheinung. Proceedings of the International Conference on Quantitative Genetics 5: 1148-1152.

Negrete, A. y J. Soberón. 1994. Los mamíferos silvestres de la reserva ecológica El Pedregal. In: Rojo, A. (ed.). Reserva Ecológica "El Pedregal” de San Ángel: Ecología, historia natural y manejo. Universidad Nacional Autónoma de 
México. México, D.F., México. Pp. 219-228.

Preuss, S. y C. S. Pikaard. 2007. rRNA gene silencing and nucleolar dominance: Insights into a chromosome-scale epigenetic on/off switch. Biochimica et Biophysica Acta Gene Structure and Expression 1769(5-6): 383-392. DOI: http://dx.doi.org/10.1016/j.bbaexp.2007.02.005

Raven, P. H. 1975. The bases of angiosperm phylogeny: cytology. Annals of the Missouri Botanical Gardens 62(3): 724-764. DOI: http://dx.doi.org/10.2307/2395272

Rodríguez, A. y K. J. Sytsma. 2006. Phylogenetics of the "Tigerflower" group (Tigridieae: Iridaceae): Molecular and morphological evidence. Aliso 22(1): 412-424.

Rojo, A. y J. Rodríguez. 2002. La flora del Pedregal de San Ángel. Instituto Nacional de Ecología-Secretaría de Medio Ambiente y Recursos Naturales (INE-SEMARNAT). México, D.F., México. 95 pp.

Rzedowski, J. 1954. Vegetación del Pedregal de San Ángel (Distrito Federal, México). Anales Escuela Nacional Ciencias Biológicas, Instituto Politécnico Nacional 8: 59129.

Sakai, B. 1952. Zytologische Untersuchungen bei Iridaceen I. Cytologia 17(2): 104-111. DOI: http://doi.org/10.1508/ cytologia.17.104

Sharma, A. K. y A. Sharma. 1961. Cytology of some members of the family Iridaceae. Cytologia 26(3-4): 274-284. DOI: http://doi.org/10.1508/cytologia.26.274

Sinha, S. S. N. y H. Roy. 1979. Cytological studies in the genus Phaseolus I. Mitotic analysis in fourteen species. Cytologia 44(1): 191-199. DOI: http://doi.org/10.1508/ cytologia.44.191

Stebbins, G. L. 1971. Chromosomal evolution in higher plants. Addison-Wesley Publishing. Reading, USA. 216 pp.

Tapia-Pastrana, F. 2014. Los rompimientos cromosómicos y translocaciones modelan el cariotipo trimodal de una po- blación hexaploide de Milla biflora (Asparagaceae) de la Reserva Ecológica del Pedregal de San Ángel, Distrito Federal, México. Revista Mexicana de Biodiversidad 85(2): 598-605. DOI: http://dx.doi.org/10.7550/rmb.40412

Tapia-Pastrana, F. y P. Mercado-Ruaro. 2001. A combination of the "squash" and "splash" techniques to obtain the karyotype and assess meiotic behavior of Prosopis laevigata L. (Fabaceae: Mimosoideae) Cytologia 66(1): 11-17. DOI: http://doi.org/10.1508/cytologia.66.11

The Plant List. 2013. Version 1.1. http://www.theplantlist. org/1.1/browse/A/Iridaceae/Tigridia/ (consultado diciembre de 2016).

UNAM. 2005. Crece la Reserva Ecológica del Pedregal de San Ángel de Ciudad Universitaria. Gaceta UNAM 3813: 14$15,19-21$.

Valiente-Banuet, A. y G. E. de Luna. 1990. Una lista florística actualizada para la reserva del Pedregal de San Ángel, México, D.F. Acta Botanica Mexicana 9: 13-30. DOI: http://dx.doi.org/10.21829/abm9.1990.588

Vázquez, G. L. M., T. H. Norman M. y M. del C. Corona. R. 2001. Oceloxóchitl Tigridia pavonia (L.f.) DC. Colección: Ciencias Naturales y Exactas, Serie: Ciencias Agrícolas. Universidad Autónoma del Estado de México. Toluca, México. 69 pp.

Velázquez-López, R. R., Z. Cano-Santana y X. DamiánDomínguez. 2009. Historia natural y biología reproductiva de la flor del tigre, Tigridia pavonia (Iridaceae). In: Lot, A. y Z. Cano-Santana (eds.). Biodiversidad del Pedregal de San Ángel. Reserva Ecológica del Pedregal de San Ángel y Coordinación de la Investigación Científica, Universidad Nacional Autónoma de México. México, D.F., México. Pp. 329-336. 